\title{
Téoros
}

Revue de recherche en tourisme

\section{Entre gloire et infamie}

\section{Le rôle ambivalent du tourisme dans la mise en valeur du Vieux-Montréal}

\section{Martin Drouin}

Volume 28, numéro 2, 2009

URI : https://id.erudit.org/iderudit/1024812ar

DOI : https://doi.org/10.7202/1024812ar

Aller au sommaire du numéro

Éditeur(s)

Université du Québec à Montréal

ISSN

0712-8657 (imprimé)

1923-2705 (numérique)

Découvrir la revue

Citer cette note

Drouin, M. (2009). Entre gloire et infamie : le rôle ambivalent du tourisme dans la mise en valeur du Vieux-Montréal. Téoros, 28(2), 93-96.

https://doi.org/10.7202/1024812ar d'utilisation que vous pouvez consulter en ligne.

https://apropos.erudit.org/fr/usagers/politique-dutilisation/ 


\title{
Entre gloire et infamie Le rôle ambivalent du tourisme dans la mise en valeur du Vieux-Montréal
}

\author{
Martin DROUIN \\ Coordonnateur, Institut du patrimoine \\ Professeur associé, Département d'études urbaines et touristiques \\ Université du Québec à Montréal \\ drouin.martin@uqam.ca
}

Dans une précédente chronique, j'ai évoqué le rôle fondamental joué par la fonction touristique dans la renaissance et la réhabilitation du Vieux-Montréal. Alors que le quartier se cherchait une vocation à la fin des années 1950, la volonté d'en faire un haut lieu de l'histoire montréalaise mis en scène pour des visiteurs étrangers permit d'inscrire le futur à l'aune d'un nouvel horizon d'attente. Le dynamisme engendré par la tenue de l'Exposition universelle de 1967 alimenta l'optimisme avec lequel les autorités municipales et les acteurs locaux s'attelèrent à la tâche. Bien sûr, l'objectif d'organiser et de proposer une offre touristique distinctive dans le Vieux-Montréal ne se fit pas sans heurts. Cependant, structurés autour de la valorisation de l'identité canadienne-française dont le «Old French Quarter» était l'écrin, les aménagements freinèrent les démolitions qui semblaient s'accumuler chaque jour davantage tout en justifiant le bien-fondé d'une telle démarche. Bref, une vision extrêmement positive du tourisme renversait l'opération prévisible de «renouveau urbain », appliquée ailleurs dans les autres quartiers anciens de la métropole (Drouin, 2009). Au cours des trois dernières décennies, une véritable rupture a complètement métamorphosé le rapport entre patrimoine et tourisme dans le même secteur. Que s'est-il donc passé? Est-il possible de mieux comprendre cette mutation?

La mise en valeur du Vieux-Montréal aurait-t-elle participé, par la valorisation d'une identité nationale matérialisée dans le patrimoine, à la consolidation d'une appropriation locale du quartier au détriment d'une mise en scène de l'autre, dont se nourrit le tourisme? Au tournant des années 1970, l'animation implantée progressivement depuis une décennie a favorisé une fréquentation nouvelle des lieux. Des galeries d'art, antiquaires et restaurants aux saveurs traditionnelles permettaient de croire à la réalisation du programme initié par les premiers promoteurs. Le Vieux-Montréal apparaissait comme un succès! À cette offre, s'ajoutait celle de bars, terrasses et boîtes à chanson qui accueillaient une jeunesse montréalaise attirée par les échos du «Vive le Québec libre!» lancé par le général De Gaulle au balcon de l'Hôtel de Ville le 24 juillet 1967. La rue Saint-Paul et surtout la place JacquesCartier, réaménagées toutes les deux en 1964, devinrent la plaque tournante de la vie culturelle et intellectuelle francophone. Il ne fut pas étonnant par exemple que le groupe Beau Dommage, digne représentant de la nouvelle génération musicale québécoise, ait donné son premier concert à l'Hôtel Nelson, en plein cœur du Vieux-Montréal. Bien d'autres manifestations s'inscrivirent dans cette dynamique. D'ailleurs, les célébrations de la fête nationale du Québec ne se déroulèrent-elles pas plusieurs années consécutives sur la place Jacques-Cartier (Gagnon, 1971; Sans auteur, 1974) ? À cet égard, le quartier s'affichait clairement comme le témoin des mutations de l'identité à l'œuvre. Un nouveau nationalisme, moderne, conquérant et sensible à la dimension urbaine de son expression, y prenait racine (Linteau et al, 1989). Le regard de l'autre devenait moins important que l'affirmation forte et revendicatrice de l'existence de soi. On pourrait y voir une première rupture de la place du tourisme dans le Vieux-Montréal.

Si le tourisme s'imposait et permettait au secteur de devenir l'une des principales attractions de Montréal, les avatars de la nouvelle fonction économique questionnaient la véritable vocation du quartier. Tout d'abord, certains commerçants étaient déçus par le caractère saisonnier de la fréquentation des visiteurs. Ils espéraient une manne dont les effets auraient été plus vigoureux et permanents. Mais surtout, le tourisme ne prenait pas toujours la forme qui avait été imaginée lorsque la réhabilitation fut lancée au début des années 1960. La corrélation entre la mise en valeur d'un lieu chargé d'histoire et l'affirmation d'un tourisme culturel s'avérait plutôt décevante. Bien sûr, comme nous venons de le voir, des événements associaient l'image du Vieux-Montréal à une activité à caractère identitaire, mais ils étaient surtout l'apanage de la population locale. La majorité des autocars qui se succédaient devant l'église Notre-Dame et sur la rue Saint-Paul empruntait davantage 


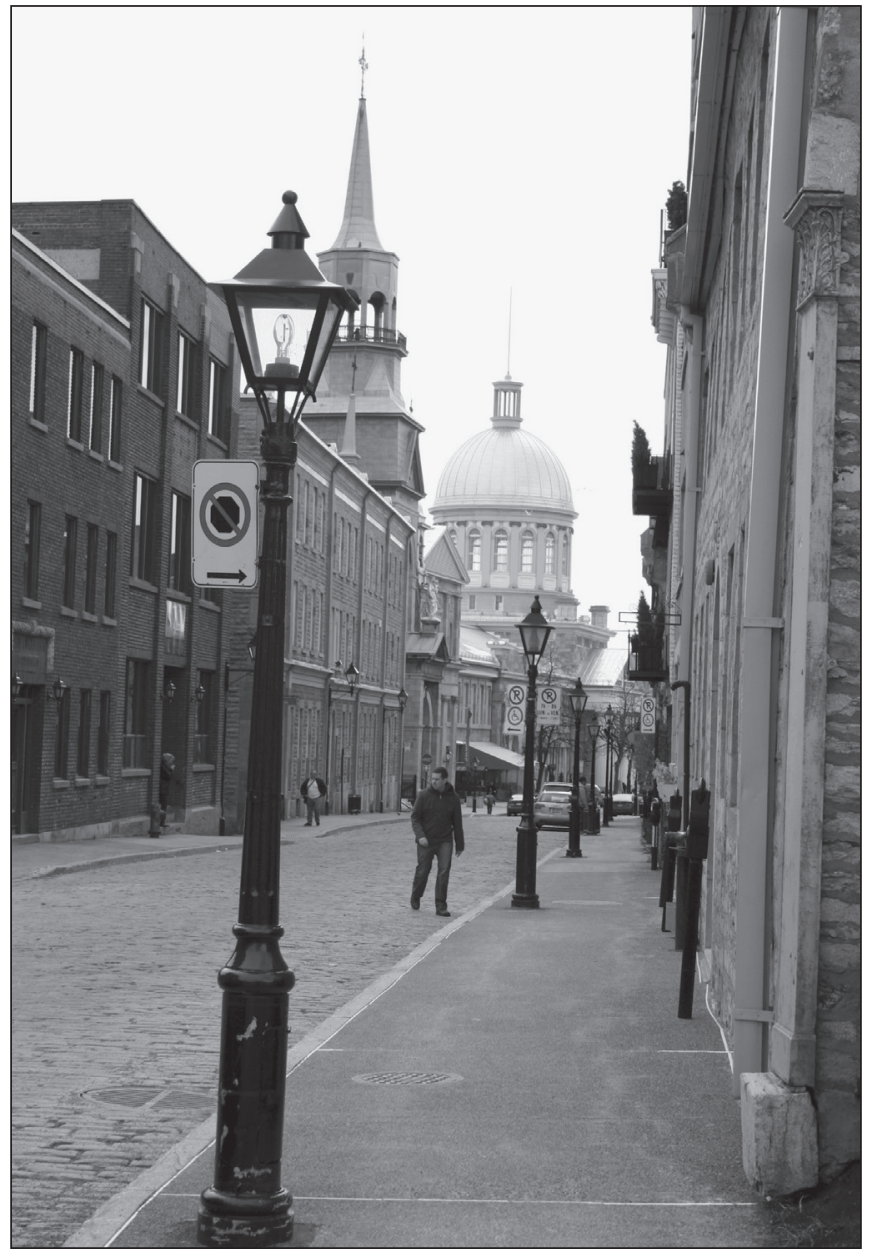

ILLUSTRATION 1 : La rue Saint-Paul

(photo : Martin Drouin).

aux caractéristiques du tourisme de masse qu'à la recherche d'une expérience authentique du lieu. Le projet de faire du Vieux-Montréal la vitrine de l'identité canadienne-française semblait être dénaturé par une consommation limitée et superficielle du quartier. En parallèle, certains dénonçaient la prolifération de vendeurs de pacotilles et autres marchandises aux accents d'Orient. Les protestataires trouvaient que l'odeur de patchouli planait bien davantage sur le Vieux-Montréal que celle du sirop d'érable. Enfin, d'autres estimaient que la partie ouest, c'est-à-dire le secteur qui s'étendait grosso modo entre le boulevard Saint-Laurent et la rue McGill, n'avait pas bénéficié des effets du nouvel usage du quartier (Drouin, 2008). L'introduction de la fonction touristique avait-elle tenu ses promesses? Tous ne semblaient pas d'accord sur la réponse à donner à cette question.

Une autre interrogation vint fragiliser la place du tourisme dans le Vieux-Montréal. Elle faisait écho aux protestations qui animaient les transformations du centre-ville. Depuis le début des années 1970, des luttes s'étaient engagées contre les démolitions engendrées par la modernisation de la métropole et pour la reconnaissance de l'architecture du $19^{\mathrm{e}}$ siècle. À mesure que

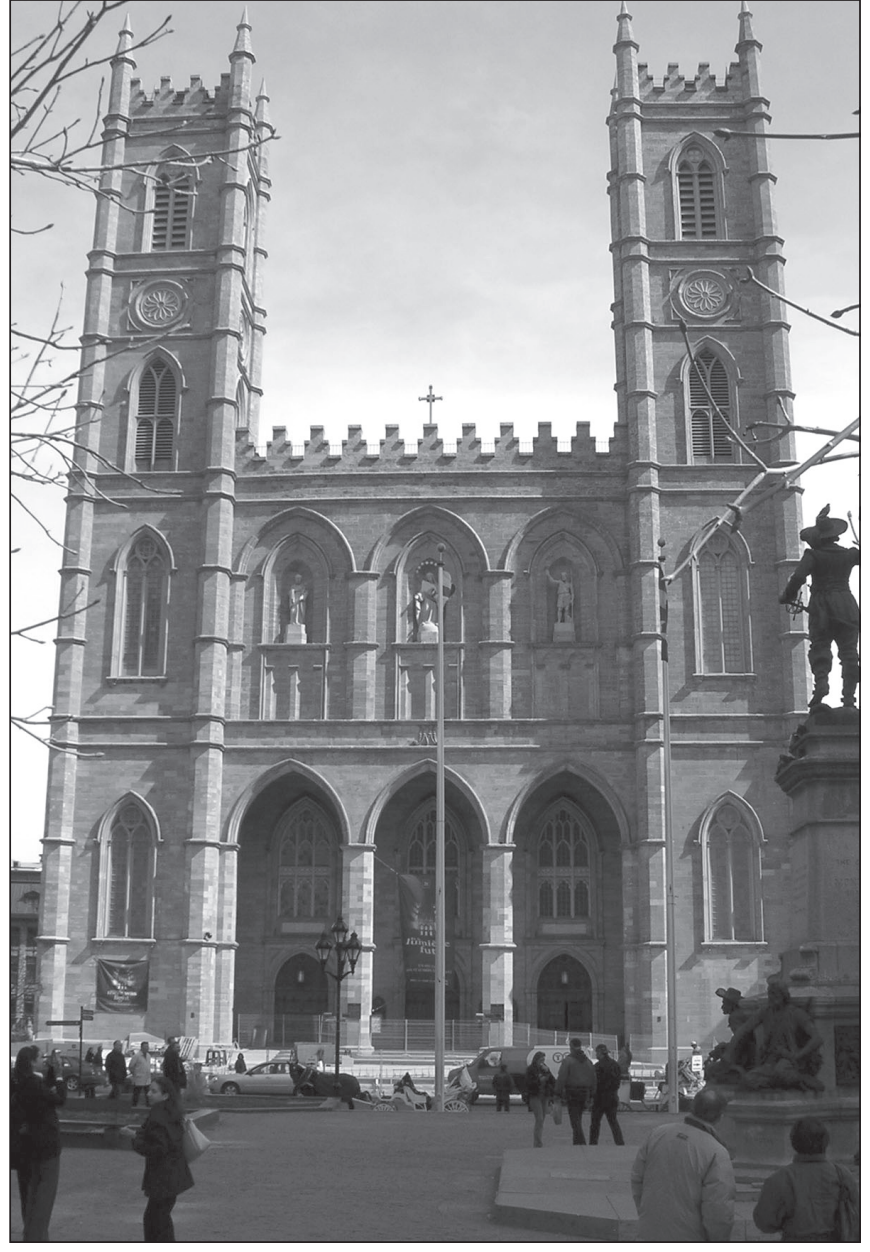

ILLUSTRATION 2 : La basilique Notre-Dame de Montréal

(photo : Martin Drouin).

la décennie avançait, le développement de l'arrondissement historique apparaissait de plus en plus apparenté à celui des quartiers centraux, non pas seulement associé à l'histoire du début de la colonie. Cette idée fut particulièrement débattue lorsque fut connue la volonté de reconstruire l'ancien hôpital général des sœurs grises, ce vaste ensemble conventuel, semblable à ce qu'il fût avant le départ des religieuses au $19^{\mathrm{e}}$ siècle. Le projet aurait pu être réalisé dans les années 1960 alors que la conception du monument historique et le désir de retrouver des édifices d'inspiration française encourageaient une telle restitution. Une dizaine d'années plus tard, il était vivement condamné au nom de la guerre du faux et de la démolition de magasins-entrepôts, situés sur la rue Saint-Pierre, dont les mérites étaient désormais proclamés (Drouin, 2005). Le spectre de la «disneylandisation» de l'arrondissement historique s'abattait sur les opérations de mise en valeur. S'inscrivant en porte-à-faux de la nouvelle fonction touristique du quartier qui aurait bénéficié de la présence de cet attrait spectaculaire, les protestataires réclamaient le retour des résidants.

Dans les années 1970, de nouvelles avenues s'offraient progressivement à la réutilisation du patrimoine (Weinberg, 
ILLUSTRATION 3 :

La rue Saint-Pierre

(photo : Martin Drouin).

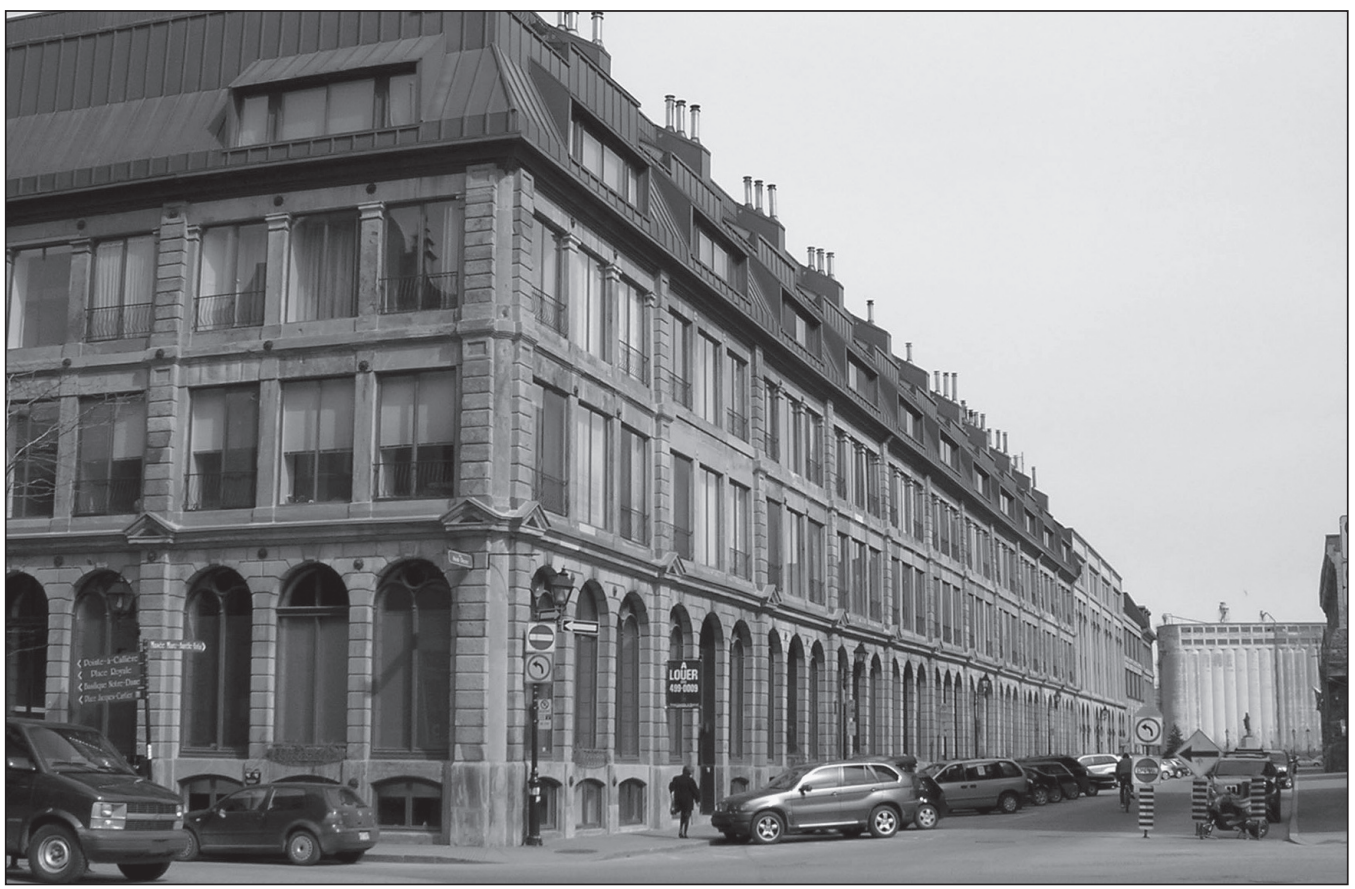

1977). Parmi celles-ci, le recyclage pour des fins résidentielles permettait une alternative viable à un usage muséal, valorisé depuis le début du 20 siècle (Coleman, 1933). Quelques pionniers avaient, dès les années 1960, démontré la possibilité de vivre dans le Vieux-Montréal. Toutefois, avec à peine cinq cents habitants au milieu de la décennie suivante, la marginalisation de la fonction s'accentuait inexorablement. Le quartier allait-il continuer de se vider de sa population pour devenir un lieu de délectation esthétique et historique? La perspective d'en faire une ville fantôme était bien réelle. C'est dans ce contexte que fut lancé le projet des cours Le Royer. Les magasins-entrepôts que les religieuses hospitalières de Saint-Joseph avaient fait construire entre 1861 et 1874 sur l'emplacement de l'ancien Hôtel-Dieu ne seraient finalement pas recyclés en un immense musée municipal, tel que le préconisait la Société historique de Montréal dans les années 1960. Un promoteur choisit plutôt d'en faire un complexe résidentiel de deux cent vingt unités. Couronné du prix d'honneur national décerné par la Fondation Héritage Canada en 1977, le projet infléchissait une tendance lourde dans le Vieux-Montréal. Au même moment, les bâtiments de la rue Saint-Pierre furent acquis par la Société canadienne d'hypothèques et de logement (SCHL), mettant ainsi fin aux menaces de démolition. L'ensemble de la rue fut converti en résidences en copropriété quelques années plus tard (SCHL, 1982). Depuis la livraison de ces appartements, la population n'a cessé de croître pour atteindre plus de trois mille personnes en 2006 (Ville de Montréal, 2009). Le rapport entre tourisme et patrimoine s'en est trouvé depuis complètement bouleversé. Le premier était accusé d'aseptiser le second au profit d'une vitalité artificielle. Dans ce nouvel équilibre, l'apport de résidants apparaissait comme la solution efficace pour créer un quartier vivant, n'abordant que très rarement la problématique de l'embourgeoisement. Le processus semblait un mal de moindre importance que l'activité touristique.

Un autre événement marqua un changement d'attitude dans le projet associé à l'avenir du Vieux-Montréal. En 1979, la Ville de Montréal et le ministère des Affaires culturelles (aujourd'hui le ministère de la Culture, des Communications et de la Condition féminine) signèrent une entente de collaboration pour assurer la sauvegarde, la réhabilitation et la mise en valeur de l'arrondissement historique. Depuis que le quartier ancien avait été protégé par le gouvernement provincial en 1964, les prérogatives entre les deux paliers de gouvernement avaient été la pierre d'achoppement sur laquelle butait le processus d'intervention. Les autorités municipales étaient peu enclines à laisser à leurs homologues québécois de trop grands pouvoirs d'ingérence. La signature de l'Entente sur le Vieux-Montréal et le patrimoine montréalais - de son nom officiel - créait un partenariat entre les deux instances à partir duquel une action concertée était désormais possible. L'entente, qui fête ses trente ans cette année après plusieurs renouvellements, a permis l'injection de plus de deux cent quatre-vingt-dix millions de dollars (Lamarche, 2004). Il serait long et fastidieux d'énumérer l'ensemble des projets réalisés. Toutefois, on peut retenir qu'elle permit une meilleure connaissance du Vieux-Montréal grâce à des inventaires systématiques et différentes études thématiques. Le patrimoine y apparaissait beaucoup plus complexe. Les témoins de la ville coloniale et préindustrielle, jusque-là le support d'une identité nationale, devaient dès lors cohabiter avec une succession de couches historiques qui avaient marqué à la fois l'histoire du quartier et celle de la métropole. Le produit touristique et les représentations associées à l'arrondissement historique ne correspondaient plus à une compréhension fine du milieu. De plus, le patrimoine, soutenu financièrement par les autorités, n'avait plus nécessairement besoin du projet 
touristique pour survivre. Au contraire, la peur de muséifier le quartier départageait désormais la culture du tourisme, le premier se présentant comme une meilleure approche de la mise en valeur du patrimoine au détriment du second. Le tourisme perdait encore une fois de son lustre.

Les célébrations entourant le $350^{\mathrm{e}}$ anniversaire de la fondation de Montréal en 1992 accentuèrent la problématique entre tourisme, résidants et patrimoine. Outre l'ouverture du musée d'archéologie et d'histoire de Montréal, Pointe-à-Callière, et de nouveaux aménagements urbains, les festivités densifièrent l'animation du quartier. Si les activités se déroulèrent sans trop de heurts, la possibilité de réitérer l'expérience l'année suivante fut un point tournant. La polémique entourant la tenue du spectacle Le Grand jeu de nuit, présenté sur la place d'Armes, représentait bien la fracture entre les deux mondes. D'un côté, des résidants jugeaient l'animation trop bruyante et dérangeante pour la vie quotidienne du quartier. De l'autre, des commentateurs, bien que pouvant comprendre la nature des récriminations, croyaient que ces derniers étaient pleinement conscients des inconvénients d'un lieu de tourisme. Le questionnement était fondamental puisqu'il mena à la tenue du Forum sur le tourisme dans le Vieux-Montréal, organisé en 1993 par la Société immobilière du patrimoine architectural de Montréal (SIMPA) et la Chaire de tourisme de l'École des sciences de la gestion de l'Université du Québec à Montréal. Les objectifs énoncés visaient à «favoriser une prise de conscience de l'activité touristique [...], à comprendre les seuils de tolérance du quartier à l'égard de cette activité, à suggérer la prise en charge par le milieu et la mise en valeur des ressources touristiques matérielles et culturelles sur la base d'un développement durable» (Bédard et Huard, 1996 : i). Une association des résidants du Vieux-Montréal fut d'ailleurs créée à cette occasion. Une table de concertation du Vieux-Montréal, mise sur pied au lendemain de la rencontre, tente, depuis, d'harmoniser les différents usages. Les efforts de dialogue et de consultation constituent clairement une singularité du quartier historique dans la recherche d'équilibre entre la conservation du patrimoine et le développement d'un tourisme à la fois respectueux de la ressource et de son milieu.

Au départ de cette chronique, le rôle ambivalent joué par le tourisme dans la mise en valeur du Vieux-Montréal était questionné. Si le président de Tourisme Montréal, Charles Lapointe, présente encore aujourd'hui l'arrondissement historique comme la locomotive du tourisme à Montréal (Lapointe, 2009), force est de constater que l'affirmation n'est pas facile à accepter par tous. Ainsi, le basculement d'un projet extrêmement positif au début des années 1960 à une position exiguë, presque inconfortable, me poussa à explorer les mutations à l'œuvre au cours des quarante dernières années. Sans prétendre à une exhaustivité du phénomène, $\mathrm{j}$ 'ai voulu identifier quelques moments clefs qui permettent de comprendre les transformations en cours dans le quartier. Dans ce processus, le rapport à l'autre, celui qui a fondé le développement du tourisme depuis le Siècle des Lumières et a permis la découverte de patrimoines régionaux, souvent insoupçonnés par la population locale, est complètement inversé. Les promoteurs du tourisme espèrent toujours une fréquentation soutenue de l'arrondissement historique par une clientèle étrangère alors que ceux du patrimoine travaillent plutôt à une appropriation des lieux par les Montréalais. Tout dépendant du point de vue où l'on se place, l'avenir du Vieux-Montréal n'est pas logé à la même enseigne. Le débat n'est pas clos même si le discours culturel semble actuellement dominant. En effet, alors que la thématisation des quartiers bat son plein à Montréal avec la création de la Cité du multimédia, du Quartier du commerce mondial, du Quartier des spectacles, du Quartier de la vie, le Vieux-Montréal continue d'être balotté entre deux pôles. Serat-il désormais connu sous le nom de Quartier de l'histoire, valorisant ainsi un choix de mise en valeur proposé depuis les années 1960 (Linteau et Burgess, 2010)? Sera-t-il plutôt un quartier «ordinaire», comme les autres de la métropole, selon le souhait de plusieurs depuis les années 1970 ? Entre gloire et infamie, la fonction touristique questionnera encore pendant plusieurs années l’identité du Vieux-Montréal.

\section{Bibliographie}

BÉDARD, François et Hélène HUARD (1996) Le tourisme et la qualité de vie dans le Vieux-Montréal, Montréal, Chaire de tourisme, École des sciences de la gestion, Université du Québec à Montréal, 35 p.

COLEMAN, Laurence V. (1933) Historic House Museums, Washington, American Association et Museums, $187 \mathrm{p}$.

DROUIN, Martin (2009) «Le tourisme dans le Vieux-Montréal : une fonction au cœur de sa renaissance et de sa réhabilitation », Téoros, vol. 28, $\mathrm{n}^{\circ} 1$, p. 93-96.

DROUIN, Martin (2008) «La renaissance du Vieux-Montréal : usage, histoire et architecture (1960-1979)», dans Capucine Lemaître et Benjamin Sabatier (dir.), Patrimoine et patrimonialisation : fabrique, usages et réemplois, Québec, Éditions MultiMondes, p. 179-202.

DROUIN, Martin (2005) Le combat du patrimoine à Montréal (1973-2003), Québec, PUQ, 386 p.

GAGNON, Serge (1971) «Le Vieux Montréal à vol de journaliste», Point de Mire, 24 juillet.

LAMARCHE, Susie (2004) 25 ans d'entente. Les gestes, les acteurs, les témoins, Montréal, Société de développement de Montréal, 24 p.

LAPOINTE, Charles (2009) «Les conditions gagnantes de Montréal», Conférence dans le cadre du Forum Urba2015, Montréal, Université du Québec à Montréal, 14 janvier, <http://www.forumurba2015. com/2_conferences/2.1_liste_conferences/2009-01-14\%20Les\%20conditions\%20gagnantes\%20de\%20Montr\%C3\%A9al.pdf>

LINTEAU, Paul-André; René DURCHER; Jean-Claude ROBERT et François RICARD (1989) Histoire du Québec contemporain, tome 2, Le Québec depuis 1930, Montréal, Boréal, 739 p.

LINTEAU, Paul-André et Joanne BURGESS (dir.) (2010) Le Vieux-Montréal, un quartier de l'histoire? Pourquoi? Comment?, Québec, Éditions MultiMondes (à paraître).

Sans auteur (1974) «Old Montreal packs 'em in», The Montreal Star, 22 juillet.

SCHL (1982) Le Cours St-Pierre : étapes de réaménagement/Le Cours St. Pierre: Redevelopment Process, Ottawa, Société canadienne d'hypothèques et de logement/Canada Mortgage and Housing Corporation, $21 \mathrm{p}$.

Ville de Montréal, Bureau du patrimoine, de la toponymie et de l'expertise (2009) Portrait socio-économique du Vieux-Montréal, site Internet officiel du Vieux-Montréal, <www.vieux.montreal.qc.ca/images/pdf/soc_econ. pdf> (Consulté le 7 septembre 2009)

WEINBERG, Nathan (1977) Preservation in American Towns and Cities, Boulder (Colorado), Westview Press, 233 p. 